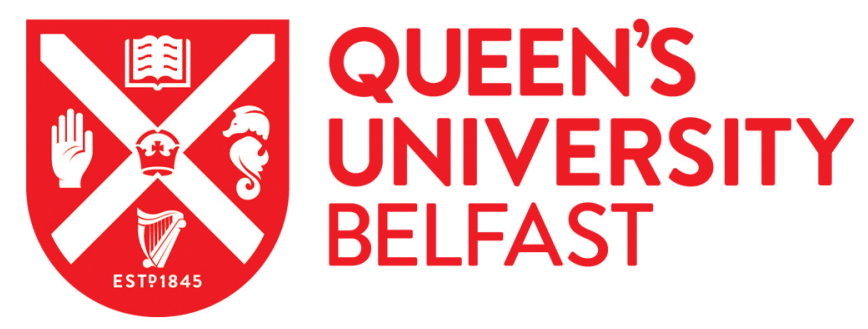

\title{
Impact of CSI Uncertainty on MCIK-OFDM: Tight, Closed-Form Symbol Error Probability Analysis
}

Luong, T. V., \& Ko, Y. (2017). Impact of CSI Uncertainty on MCIK-OFDM: Tight, Closed-Form Symbol Error Probability Analysis. IEEE Transactions on Vehicular Technology, 67(2), 1272-1279.

https://doi.org/10.1109/TVT.2017.2753402

Published in:

IEEE Transactions on Vehicular Technology

Document Version:

Peer reviewed version

Queen's University Belfast - Research Portal:

Link to publication record in Queen's University Belfast Research Portal

Publisher rights

๑) 2017 IEEE This work is made available online in accordance with the publisher's policies. Please refer to any applicable terms of use of the publisher.

\section{General rights}

Copyright for the publications made accessible via the Queen's University Belfast Research Portal is retained by the author(s) and / or other copyright owners and it is a condition of accessing these publications that users recognise and abide by the legal requirements associated with these rights.

Take down policy

The Research Portal is Queen's institutional repository that provides access to Queen's research output. Every effort has been made to ensure that content in the Research Portal does not infringe any person's rights, or applicable UK laws. If you discover content in the Research Portal that you believe breaches copyright or violates any law, please contact openaccess@qub.ac.uk. 


\title{
Impact of CSI Uncertainty on MCIK-OFDM: Tight, Closed-Form Symbol Error Probability Analysis
}

\author{
Thien Van Luong, Student Member, IEEE, and Youngwook Ko, Member, IEEE
}

\begin{abstract}
This paper proposes a novel framework to analyze the symbol error probability (SEP) for multicarrier index keying - orthogonal frequency division multiplexing (MCIK-OFDM) systems. Considering two different types of detections such as the maximum likelihood (ML) and low-complexity greedy detectors (GD), we derive tight, closed-form expressions for the average SEPs of MCIK-OFDM in presence of channel state information (CSI) uncertainty. We undertake an asymptotic performance analysis with respect to three CSI conditions, which ensures to provide a comprehensive insight into the achievable diversity and coding gains as well as the impact of various CSI uncertainties on the SEP performance. The SEP performance comparison between the ML and GD, is obtained under different CSI uncertainties. This interestingly reveals that the GD can achieve nearly optimal error performance as the $M$-ary modulation size is large, or even outperforms the ML under certain CSI conditions. Finally, the theoretical and asymptotic analysis are verified via simulation results, obtaining the high accuracy of the derived SEP.
\end{abstract}

Index Terms-MICK-OFDM, OFDM-IM, index modulation, symbol error probability, imperfect CSI, greedy detection.

\section{INTRODUCTION}

MCIK-OFDM is an emerging key technique for the next generation wireless networks due to its high spectral and energy efficiency [1]. Unlike conventional OFDM, MCIKOFDM, also termed as OFDM-IM [2], activates only a subset of sub-carriers to carry information bits through both the $M$-ary constellation symbols and the indices of active subcarriers. As a result, MCIK-OFDM requires less modulators and demodulators than the classical OFDM, which allows to reduce the complexity of transceiver structure. In addition, MCIK-OFDM can provide an attractive trade-off between the error performance and spectral efficiency by adjusting the number of active sub-carriers [2]. Hence, MCIK-OFDM is considered not only for high speed wireless communication systems, but also for machine type communications (MTC), which requires high energy efficiency at a low complexity.

MCIK-OFDM with the flexible number of active subcarriers was first introduced in [2]. Various concepts of MCIKOFDM have been studied in [2]-[24]. Recently, in [3], a tight bound on the bit error rate (BER) is derived, while the achievable rate is investigated in [4]. A generalized version of MCIK is proposed in [5], where the number of active subcarriers is no longer fixed. In [6], a low-complexity greedy detector (GD) based on energy detection is proposed, and then this detector is analyzed under the generalized fading in [7]. A number of techniques that aim at improving the

Copyright (c) 2015 IEEE. Personal use of this material is permitted. However, permission to use this material for any other purposes must be obtained from the IEEE by sending a request to pubs-permissions@ieee.org. The authors are with the ECIT Institute, Queen's University Belfast, Belfast, BT3 9DT United Kingdom, e-mail: \{tluong01, y.ko\}@qub.ac.uk. error performance and diversity gain can be found in [8][11], while several solutions to enhance the spectral efficiency are proposed in [12]-[14]. Very recently, the extension of MCIK to multiple antenna systems is introduced with the maximum likelihood (ML) detection in [15], and its lowcomplexity detectors is proposed in [16]. The MCIK-OFDM with hybrid low complexity greedy detection and multiple antenna diversity reception is proposed in [17]. The detailed survey of IM techniques can be found in [18], [19]

It is noteworthy that most of existing works focus on the BER performance analysis for the ML detection [20]-[22], or for the energy detectors like the GD [23], [24], while little work on the SEP analysis for MCIK-OFDM has been done. In fact, existing expressions for the BER are not able to provide a comprehensive insight into the achievable diversity order and coding gain of system. Especially, impacts of MCIKOFDM parameters such as the number of active sub-carriers on the performance has not been fully addressed. Furthermore, effects of the channel state information (CSI) uncertainty on the error probability of MCIK-OFDM using either the ML or GD has not been investigated in the literature. In [17], the SEP expression is first derived, for MCIK-OFDM with only the GD and under perfect CSI. This expression is not tight even in high SNR regions. To the best of our knowledge, impacts of CSI uncertainty on the SEP has not been investigated, in terms of generalized MCIK-OFDM using both the ML and the GD.

In this work, we investigate a framework to evaluate the SEP of MCIK-OFDM under various CSI uncertainties. Main contributions of the paper are summarized as follows.

- We propose a new framework to analyze the average SEP for a generalized index modulation scheme. We theoretically prove that using the ML and the GD, the SEP can be split into two parts: the index error probability (IEP) and the SEP of $M$-ary complex symbols.

- Using this framework, we show that the difference on the SEP between the ML and the GD is determined only by the IEP. For this, we provide new tight, closed-form expressions for average SEPs of the ML and the GD.

- In presence of CSI uncertainty, we further analyze the impact of various types of CSI uncertainties on the average SEP. For this, the closed-form expression for the generalized SEP is derived under fixed and variable minimum mean square error (MMSE) based channel estimation errors. This ensures to provide a comprehensive insight into the impact of CSI uncertainty.

- The transmit diversity and coding gains are theoretically analyzed in terms of the index activation and various CSI conditions. To the best of our knowledge, the relationship between the transmit diversity (and coding gain) of index modulation and the CSI uncertainty has not been reported 
in the MCIK-OFDM systems.

- Simulation results verify the accuracy of our theoretical and asymptotic analysis for MCIK-OFDM using the ML and the GD, under three CSI conditions. Interestingly, the SEP of the GD is shown to be less sensitive to the CSI uncertainty, compared to the ML. This reveals that for given CSI uncertainty, the GD even at low complexity can offer better error performance than the ML.

The rest of this paper is organized as follows. Section II presents the MCIK-OFDM system model with both the ML and GD detectors. The closed-form expressions for the average SEP of the ML and GD, taking into account imperfect CSI are derived in Section III. In Section IV, we present the asymptotic performance analysis under various CSI conditions. Section V presents simulation results. Section VI concludes the paper.

Notation: Lower-case bold and upper-case bold letters represent vectors and matrices, respectively. $C($,$) is the binomial$ coefficient and $\lfloor$.$\rfloor is the floor function. \mathfrak{R e}\{$.$\} and \mathfrak{I m}\{$. denote the real and imaginary parts of a complex number, respectively. $\mathcal{C N}($,$) denotes the complex Gaussian distribution.$ The expectation operator and the moment generating function (MGF) are presented by $\mathbb{E}\{$.$\} and \mathcal{M}\{$.$\} , respectively.$

\section{SySTEM MODEL}

\section{A. MCIK-OFDM System Model}

Consider an MCIK-OFDM system where a transmitter node communicates to a receiver node over $N_{c}$ sub-carriers in total, including $G$ groups of $N$ sub-carriers, i.e., $N_{c}=G N$. In every MCIK-OFDM transmission, only $K$ out of $N$ sub-carriers per group are activated, while $N-K$ remaining sub-carriers are zero padded. Employing the MCIK-OFDM operation independently to each group, without loss of generality, we focus on addressing only one group.

For each group, the MCIK-OFDM transmission consists of two bit streams ( $p_{1}$ and $p_{2}$ bits). The first $p_{1}$ bits are mapped to a possible combination of $K$ active sub-carriers. Denote a combination of $K$ active indices by $\theta=\left\{\alpha_{1}, \ldots, \alpha_{K}\right\}$, where $\alpha_{k} \in\{1, \ldots, N\}$ for $k=1, \ldots, K$. This combination is referred to as index symbol, being determined by $p_{1}$ bits. The second $p_{2}$ bits are mapped to $K$ complex $M$-ary symbols, which is denoted by $\mathbf{s}=\left[s\left(\alpha_{1}\right), \ldots, s\left(\alpha_{K}\right)\right]$, where $s\left(\alpha_{k}\right) \in \mathcal{S}, k=$ $1, \ldots, K$, and $\mathcal{S}$ denotes the $M$-ary constellation. Utilizing both $\theta$ and $\mathbf{s}$, the transmitted MCIK-OFDM codeword is generated as $\mathbf{x}=[x(1), \ldots, x(N)]^{T}$, where $x(\alpha)=s(\alpha)$ for $\alpha \in \theta$ and $x(\alpha)=0$ for $\alpha \notin \theta, \alpha=1, \ldots, N$. Notice that for given $N$ and $K$, we have $p_{1}=\left\lfloor\log _{2} C(N, K)\right\rfloor$ and $p_{2}=K \log _{2} M$. Thus, for each group of MCIK-OFDM, the total number of data bits per transmission is $p=\left\lfloor\log _{2} C(N, K)\right\rfloor+K \log _{2} M$.

The received signal in the frequency domain is written as

$$
\mathbf{y}=\mathbf{H} \mathbf{x}+\mathbf{n},
$$

where $\mathbf{H}=\operatorname{diag}\{h(1), \ldots, h(N)\}$ is the channel matrix, $h(\alpha)$ denote the Rayleigh fading channel coefficient of sub-carrier $\alpha$, satisfying $h(\alpha) \sim \mathcal{C N}(0,1)$, and $\mathbf{n}=$ $[n(1), \ldots, n(N)]^{T}$ is the additive white Gaussian noise with $n(\alpha) \sim \mathcal{C N}\left(0, N_{0}\right)$. For each sub-carrier, the transmit power of non-zero symbols is given as $\varphi E_{s}$, where $E_{s}$ and $\varphi=N / K$ are the average transmit power of the $M$-ary symbol and the power allocation coefficient, respectively. Thus, $\bar{\gamma}=\varphi E_{s} / N_{0}$ denotes the SNR per active sub-carrier.

\section{B. Imperfect CSI Based Receiver}

We consider a practical system that suffers from the uncertain estimation of CSI at the receiver. The estimate of $h(\alpha)$ is denoted by $\hat{h}(\alpha)$, satisfying

$$
h(\alpha)=\hat{h}(\alpha)+e(\alpha),
$$

where $e(\alpha)$ is the channel estimation error, and $e(\alpha) \sim$ $\mathcal{C N}\left(0, \epsilon^{2}\right), \hat{h}(\alpha) \sim \mathcal{C N}\left(0,1-\epsilon^{2}\right)$, where $\epsilon^{2}$ denotes the CSI estimation error variance.

For data detection, either the ML or the GD is employed to decode transmitted signal in the presence of imperfect $\hat{h}(\alpha)$.

1) Maximum Likelihood Detection: The ML detector for MCIK-OFDM under the imperfect CSI can compute

$$
\hat{\mathbf{x}}=\arg \min _{\mathbf{x}}\|\mathbf{y}-\hat{\mathbf{H}} \mathbf{x}\|^{2},
$$

where $\hat{\mathbf{x}}$ is the estimated signal and is used to recover

the index symbol $\hat{\theta}$ and $K$ complex $M$-ary symbols $\hat{\mathbf{s}}$.

2) Greedy Detection (GD): The GD scheme has two separate steps as follows. In the first step, GD estimates $K$ active indices, i.e., $\hat{\theta}=\left\{\hat{\alpha}_{1}, \ldots, \hat{\alpha}_{K}\right\}$, corresponding to $K$ greatest received energies $|y(\alpha)|^{2}$.

In the next step, non-zero $M$-ary symbols are individually detected by using ML decision only on estimated active subcarriers $\hat{\alpha}$, for $\hat{\alpha} \in \hat{\theta}$. For given $\hat{\alpha}$ and $\hat{\theta}$, we compute

$$
\hat{x}(\hat{\alpha})=\arg \min _{x(\hat{\alpha}) \in \mathcal{S}}|y(\hat{\alpha})-\hat{h}(\hat{\alpha}) x(\hat{\alpha})|^{2} .
$$

Notice that the GD has a much lower complexity than the ML, at the cost of an affordable loss of reliability.

\section{SEP ANALYSIS WITH UNCERTAIN CSI}

We now analyze the SEP of MCIK-OFDM in the presence of uncertain CSI, taking into account two types of detector schemes. For this, we investigate new closed-form, accurate expressions for the SEP of MCIK-OFDM as follows.

\section{A. SEP Definition of MCIK-OFDM}

In fact, an accurate closed-form expression for the SEP of an index modulation scheme like MCIK-OFDM has not been done in the literature, even not for perfect CSI. This motivates us to introduce a novel definition of the symbol error event for MCIK-OFDM. Relying on this, a new approach is proposed to derive tight SEP expressions for both the ML and GD, moreover, taking CSI uncertainty into consideration.

First, notice that one MCIK-OFDM codeword $\boldsymbol{x}$ contains $K+1$ symbols, which are $K$ non-zero $M$-ary symbols $x(\alpha)$ for $\alpha \in \theta$ and one index symbol $\theta$. A symbol error event occurs if any of the $K+1$ symbols are incorrectly detected. That is, for each transmitted codeword $x$, there are at most $K+1$ symbols in error events. Based on this definition, the instantaneous SEP (denoted by $P_{s}$ ), can be formulated as

$$
P_{s}=\frac{P_{I}+K P_{D}}{K+1},
$$


where $P_{I}$ is the instantaneous index error probability (IEP) that $\theta$ is incorrectly detected and $P_{D}$ denotes the instantaneous error probability of $M$-ary symbols. The detailed definition of these probabilities is explained in the following.

Denote by $P_{I}(\alpha)$ the probability that the active sub-carrier $\alpha$ is detected as an inactive one. For given $N, K$ and active subcarrier $\alpha$, using the union bound, we obtain

$$
P_{I}(\alpha) \leq \sum_{\tilde{\alpha} \neq \alpha=1}^{N-K} P(\alpha \rightarrow \tilde{\alpha})
$$

where $P(\alpha \rightarrow \tilde{\alpha})$ is the pairwise index error probability (PEP), that an active sub-carrier $\alpha$ is incorrectly decoded as inactive sub-carrier $\tilde{\alpha} \neq \alpha$. Thus, from (6) and the total probability theory, we have

$$
P_{I} \leq \sum_{\alpha=1}^{N} P(\alpha) P_{I}(\alpha)
$$

where $P(\alpha)=K / N$ is the probability that sub-carrier $\alpha$ is active at the transmitter.

Based on (6) and (7), $P_{D}$ can be obtained with respect to $P_{I}(\alpha)$. Particularly, provided that the index $\alpha$ is correctly detected, the probability of the mis-detection of $x(\alpha)$ equals the $M$-ary symbol error probability, $P_{M}(\alpha)$. Whereas, if $\alpha$ is incorrectly detected as an inactive one $\tilde{\alpha}$, the detector has to estimate $x(\alpha)$ from the use of a random sub-channel, i.e., $h(\tilde{\alpha})$. This leads to that the probability of the mis-detection of $x(\alpha)$ is $\frac{M-1}{M}$. Therefore, we obtain

$$
\begin{aligned}
P_{D} & =\frac{1}{N} \sum_{\alpha=1}^{N}\left[\frac{M-1}{M} P_{I}(\alpha)+\left(1-P_{I}(\alpha)\right) P_{M}(\alpha)\right] \\
& \leq \frac{1}{N} \sum_{\alpha=1}^{N}\left[\frac{M-1}{M} P_{I}(\alpha)+P_{M}(\alpha)\right]
\end{aligned}
$$

where $P_{M}(\alpha)$ is the SEP of classical $M$-ary symbol detection.

Substituting (8) and (7) into (5), finally, the instantaneous SEP in presence of CSI uncertainty and for a generalized detection can be obtained as

$$
P_{s} \leq \frac{P_{I}\left(2-\frac{1}{M}\right)+\frac{K}{N} \sum_{\alpha=1}^{N} P_{M}(\alpha)}{K+1},
$$

and its average is provided, by taking expectation of (9), as

$$
\bar{P}_{s} \leq \frac{\bar{P}_{I}\left(2-\frac{1}{M}\right)+K \bar{P}_{M}}{K+1}
$$

where $\bar{P}_{I}$ and $\bar{P}_{M}$ are the averages of $P_{I}$ and $P_{M}(\alpha)$.

Notice from (10) that $\bar{P}_{M}$ is independent of the selection of two detector types (ML and GD), while $\bar{P}_{I}$ relies on the detector type used. This reveals that the difference of the SEP between the two detectors is shown only via $\bar{P}_{I}$. Interestingly, this novel observation allows to derive tight bounds on the SEPs for both the ML and GD in a wide range of SNRs.

In the next two subsections, we will derive the IEPs and the SEPs of both the GD and ML, based on (10). For this, we assume the $M$-ary PSK modulation is employed, and we introduce the following Lemma on $\bar{P}_{M}$ for further analysis.
Lemma 1. The average SEP of classical $M$-ary PSK under CSI uncertainty having error variance $\epsilon^{2}$ is approximated by

$$
\bar{P}_{M} \approx \frac{\xi}{12}\left[\frac{1}{1+\frac{\left(1-\epsilon^{2}\right) \bar{\gamma} \rho}{1+\bar{\gamma} \epsilon^{2}}}+\frac{3}{1+\frac{4\left(1-\epsilon^{2}\right) \bar{\gamma} \rho}{3+3 \bar{\gamma} \epsilon^{2}}}\right]
$$

where $\rho=\sin ^{2}(\pi / M), \xi=1,2$ for $M=2$ and $M>2$, respectively.

Proof: See Appendix A.

\section{B. SEP of ML Detection With CSI Uncertainty}

We first analyze the IEP for the ML with CSI uncertainty. We compute the PEP, i.e., $P(\alpha \rightarrow \tilde{\alpha})$ in (6). Based on (2), the received signals at both active and inactive sub-carriers are given by $y(\alpha)=x(\alpha) \hat{h}(\alpha)+\tilde{n}(\alpha)$ and $y(\tilde{\alpha})=n(\tilde{\alpha})$, respectively, where $\tilde{n}(\alpha)=x(\alpha) e(\alpha)+n(\alpha)$ is the noise caused by the CSI uncertainty and the additive noise, i.e., $\tilde{n}(\alpha) \sim \mathcal{C N}\left(0, \varphi E_{s} \epsilon^{2}+N_{0}\right)$. Using ML criterion, the conditioned PEP on $\hat{h}(\alpha)$ and $\hat{h}(\tilde{\alpha})$ can be computed as

$$
\begin{aligned}
P(\alpha \rightarrow \tilde{\alpha}) & =P\left\{|y(\alpha)-x(\alpha) \hat{h}(\alpha)|^{2}+|y(\tilde{\alpha})|^{2}\right. \\
& \left.>|y(\alpha)|^{2}+|y(\tilde{\alpha})-x(\alpha) \hat{h}(\tilde{\alpha})|^{2}\right\} \\
& =P\{\mathfrak{R e}\{x(\alpha) \hat{h}(\tilde{\alpha}) n(\tilde{\alpha})-x(\alpha) \hat{h}(\alpha) \tilde{n}(\alpha)\} \\
& \left.>\varphi E_{s}\left[|\hat{h}(\alpha)|^{2}+|\hat{h}(\tilde{\alpha})|^{2}\right] / 2\right\} .
\end{aligned}
$$

Notice that $\mathfrak{R e}\{x(\alpha) \hat{h}(\tilde{\alpha}) n(\tilde{\alpha})-x(\alpha) \hat{h}(\alpha) \tilde{n}(\alpha)\}$ in (12) is Gaussian distributed with zero mean and variance $\varphi E_{s}\left(N_{0}+\varphi E_{s} \epsilon^{2}\right)|\hat{h}(\tilde{\alpha})|^{2}+\varphi E_{s} N_{0}|\hat{h}(\alpha)|^{2}$. Hence, after simple manipulation from (12), we obtain

$P(\alpha \rightarrow \tilde{\alpha})=Q\left[\sqrt{\frac{\bar{\gamma}\left(\hat{\nu}_{\alpha}+\hat{\nu}_{\tilde{\alpha}}\right)}{2\left(1+\frac{\bar{\gamma} \epsilon^{2} \hat{\nu}_{\alpha}}{\hat{\nu}_{\alpha}+\hat{\nu}_{\tilde{\alpha}}}\right)}}\right] \approx Q\left[\sqrt{\frac{\bar{\gamma}\left(\hat{\nu}_{\alpha}+\hat{\nu}_{\tilde{\alpha}}\right)}{2+\bar{\gamma} \epsilon^{2}}}\right]$

where $\hat{\nu}_{\alpha}=|\hat{h}(\alpha)|^{2}, \hat{\nu}_{\tilde{\alpha}}=|\hat{h}(\tilde{\alpha})|^{2}$ and we take an approximation as $\hat{\nu}_{\alpha} /\left(\hat{\nu}_{\alpha}+\hat{\nu}_{\tilde{\alpha}}\right) \approx 1 / 2$ to simplify $P(\alpha \rightarrow \alpha)$ expression.

Let $\Theta=2 \bar{\gamma} /\left(2+\bar{\gamma} \epsilon^{2}\right)$. Using the approximation of Qfunction in (40), (13) can be rewritten as

$$
P(\alpha \rightarrow \tilde{\alpha}) \approx \frac{1}{12} e^{-\frac{\Theta\left(\hat{\nu}_{\alpha}+\hat{\nu}_{\tilde{\alpha}}\right)}{4}}+\frac{1}{4} e^{-\frac{\Theta\left(\hat{\nu}_{\alpha}+\hat{\nu}_{\tilde{\alpha}}\right)}{3}} .
$$

Consequently, utilizing (6), (7) and (14), the instantaneous IEP (denoted by $P_{I_{1}}$ ) with the ML can be approximated by

$$
P_{I_{1}} \approx \frac{K}{N} \sum_{\alpha=1}^{N} \sum_{\tilde{\alpha} \neq \alpha=1}^{N-K}\left[\frac{1}{12} e^{-\frac{\Theta\left(\hat{\nu}_{\alpha}+\hat{\nu}_{\tilde{\alpha}}\right)}{4}}+\frac{1}{4} e^{-\frac{\Theta\left(\hat{\nu}_{\alpha}+\hat{\nu}_{\tilde{\alpha}}\right)}{3}}\right] .
$$

Let $\hat{\nu}_{\Sigma}=\hat{\nu}_{\alpha}+\hat{\nu}_{\tilde{\alpha}}$. The moment generating function (MGF) of $\hat{\nu}_{\Sigma}$ can be obtained by $\mathcal{M}_{\hat{\nu}_{\Sigma}}(s)=\mathcal{M}_{\hat{\nu}}^{2}(s)=$ $\left[1-\left(1-\epsilon^{2}\right) s\right]^{-2}$, where $\mathcal{M}_{\hat{\nu}}(s)=\left[1-\left(1-\epsilon^{2}\right) s\right]^{-1}$ is 
the MGF of $\hat{\nu}$. Applying the MGF approach, the closed-form expression for the average IEP in (15) can be attained as

$\bar{P}_{I_{1}} \approx \frac{\Psi_{1}}{12}\left\{\left[1+\frac{\left(1-\epsilon^{2}\right) \bar{\gamma}}{4+2 \bar{\gamma} \epsilon^{2}}\right]^{-2}+3\left[1+\frac{2\left(1-\epsilon^{2}\right) \bar{\gamma}}{6+3 \bar{\gamma} \epsilon^{2}}\right]_{(16)}^{-2}\right\}$

where $\Psi_{1}=K(N-K)$. Interestingly, it is worth noting from (16) that the average IEP is less affected by the modulation order $M$, while being determined mainly by $N$ and $K$.

Finally, plugging (16) and (11) into (10), the average SEP of the ML for given CSI uncertainty of $\epsilon^{2}$ can be

$$
\begin{aligned}
\bar{P}_{s_{1}} & \approx \frac{\tilde{\Psi}_{1}}{12}\left\{\left[1+\frac{\left(1-\epsilon^{2}\right) \bar{\gamma}}{4+2 \bar{\gamma} \epsilon^{2}}\right]^{-2}+3\left[1+\frac{2\left(1-\epsilon^{2}\right) \bar{\gamma}}{6+3 \bar{\gamma} \epsilon^{2}}\right]^{-2}\right\} \\
& +\frac{\Psi_{2}}{12}\left[\frac{1}{1+\frac{\left(1-\epsilon^{2}\right) \bar{\gamma} \rho}{1+\bar{\gamma} \epsilon^{2}}}+\frac{3}{1+\frac{4\left(1-\epsilon^{2}\right) \bar{\gamma} \rho}{3+3 \bar{\gamma} \epsilon^{2}}}\right]
\end{aligned}
$$

where $\tilde{\Psi}_{1}=(2-1 / M) \Psi_{1} /(K+1)$ and $\Psi_{2}=$ $\xi K /(K+1)$.

It can be shown in (17) that for given $N$ and $\bar{\gamma}$, the average SEP relies on both $K$ and $\epsilon^{2}$. For instance, as $K$ increases to $N, \bar{P}_{s_{1}}$ will be dominated by the second term, which reduces to the classical OFDM. Furthermore, for given $N$, the smaller $K$, the lower $\bar{P}_{s_{1}}$ can be in the presence of $\epsilon^{2}$.

\section{SEP of GD Detection With CSI Uncertainty}

As for the IEP of the GD, notice the fact that the GD detects the active sub-carrier indices without the CSI knowledge. This leads to that the instantaneous IEP is independent of the imperfect CSI $\hat{h}(\alpha)$, and thus we have [17]

$$
P_{I_{2}} \leq \frac{K}{N} \sum_{\alpha=1}^{N} \sum_{i=1}^{N-K} \frac{(-1)^{i+1} C(N-K, i)}{i+1} e^{-\frac{i \bar{\gamma} \nu_{\alpha}}{i+1}},
$$

where $\nu_{\alpha}=|h(\alpha)|^{2}$. Because of the system model, the MGF of $\nu_{\alpha}$ is given by $\mathcal{M}_{\nu}(s)=(1-s)^{-1}$. Using the MGF method to (18), we can obtain the average IEP of the GD detector, even with uncertain CSI, as

$$
\bar{P}_{I_{2}} \leq K \sum_{i=1}^{N-K} \frac{(-1)^{i+1} C(N-K, i)}{i+1+i \bar{\gamma}}
$$

As observed from (19), it is noteworthy that the GD detection attains the average IEP independent of $\epsilon^{2}$ as well as $M$.

Substituting (19) and (11) into (10), the average SEP of the GD can be expressed in the closed-form as

$$
\begin{aligned}
\bar{P}_{s_{2}} & \leq \frac{K\left(2-\frac{1}{M}\right)}{K+1} \sum_{i=1}^{N-K} \frac{(-1)^{i+1} C(N-K, i)}{i+1+i \bar{\gamma}} \\
& +\frac{\Psi_{2}}{12}\left[\frac{1}{1+\frac{\left(1-\epsilon^{2}\right) \bar{\gamma} \rho}{1+\bar{\gamma} \epsilon^{2}}}+\frac{3}{1+\frac{4\left(1-\epsilon^{2}\right) \bar{\gamma} \rho}{3+3 \bar{\gamma} \epsilon^{2}}}\right],
\end{aligned}
$$

where $\tilde{\Psi}_{1}$ and $\Psi_{2}$ are given in (17).

Interestingly, as shown from (20), the error variance $\epsilon^{2}$ appears only in $\bar{P}_{M}$. This observation reveals that the average SEP of the GD can be less sensitive to the channel estimation error than the ML. This behavior will be verified in the asymptotic analysis as well as simulation results.

\section{IMPACT OF CSI UNCERTAINTY}

To gain an insight into the impact of the channel uncertainty, we asymptotically investigate the achievable coding and diversity gains at high SNRs, for both the ML and GD detectors. Particularly, three different CSI uncertainties will be considered: perfect, fixed and variable CSI uncertainty.

Denote by $d$ and $c$ the diversity order and the coding gain, respectively. Without loss of generality, for each CSI uncertainty, we refer to the approximate average error probability in terms of $d$ and $c$, at high SNRs, as follows

$$
\bar{P}_{s} \approx\left(c \gamma_{0}\right)^{-d}
$$

where $\gamma_{0}=E_{s} / N_{0}$ be the average SNR per sub-carrier.

\section{A. Perfect CSI $\left(\epsilon^{2}=0\right)$}

Refering to (17) and (20), at high SNRs, we can obtain the asymptotic SEPs of both the ML and the GD when $\epsilon^{2}=0$, as

$$
\begin{gathered}
\bar{P}_{s_{1}} \approx \frac{13 \xi K^{2}}{48 \rho N(K+1)}\left(\frac{1}{\gamma_{0}}\right), \\
\bar{P}_{s_{2}} \approx \frac{K^{2}}{N(K+1)}\left(\omega+\frac{13 \xi}{48 \rho}\right)\left(\frac{1}{\gamma_{0}}\right),
\end{gathered}
$$

where $\omega=\left(2-\frac{1}{M}\right) \sum_{i=1}^{N-K}(-1)^{i+1} C(N-K, i) / i$.

From (22) and (23), the achievable coding gain from the use of either the ML or GD can be given by

$$
\begin{aligned}
& c_{1}=\frac{48 \rho N(K+1)}{13 \xi K^{2}}, \\
& c_{2}=\frac{N(K+1)}{K^{2}\left(\omega+\frac{13 \xi}{48 \rho}\right)} .
\end{aligned}
$$

As for the classical OFDM, the coding gain can be obtained by employing $K=N$, as

$$
c_{0}=\frac{48 \rho}{13 \xi}
$$

Based on these results, several important remarks and theorems can be drawn as follows:

Remark 1. Both MCIK-OFDM using either the ML or GD and the classical OFDM have the same diversity order $d=$ 1. The difference in the error performance among the three schemes is decided by the coding gains, i.e., $c_{0}, c_{1}, c_{2}$. Thus, only evaluating the coding gains, we are able to compare the performance gain over these schemes.

Remark 2. It can be seen from (22) that $\bar{P}_{s_{1}}$ only depends on the term related to $\bar{P}_{M}$ at high SNRs. Thus, the error performance of MCIK-OFDM with the ML is affected mainly by the $M$-ary symbol detection error instead of the index detection error. Unlike the ML, (23) shows that the SEP of MCIK-OFDM with the GD relies on the accuracy of both the index and $M$-ary symbols detections, even at high SNRs.

Corollary 1. For given $N, K$ and $M, M C I K-O F D M$ using the ML detection can outperform the classical OFDM at high SNRs with a coding gain of

$$
g=10 \log \left[\frac{N(K+1)}{K^{2}}\right](\mathrm{dB}) .
$$


Proof: The coding gain $g$ can be obtained by $g=$ $10 \log _{10}\left(c_{1} / c_{0}\right)$ with $c_{1}, c_{0}$ given in (24) and (26).

Remark 3. Using Corollary 1, we can see that when $K$ decreases, the coding gain of MCIK-OFDM increases over the OFDM. In other words, selecting a small $K$ in MCIKOFDM offers a better error performance at a certain loss in spectral efficiency, under the perfect CSI.

Theorem 1. Consider the MCIK-OFDM. For large $M(M=$ 16), the average SEP of the GD are nearly the same as that of the $M L$, i.e., $\bar{P}_{s_{2}} \approx \bar{P}_{s_{1}}$. On the other hand, for small $M$ as $(M=2,4)$, the SEP of the ML significantly outperforms that of the $G D$, i.e., $\bar{P}_{s_{1}} \ll \bar{P}_{s_{2}}$.

Proof: Based on the observation of $\omega$ and $13 \xi / 48 \rho$ in (22) and (23), as $M \geq 16$, we have $13 \xi / 48 \rho \gg \omega$, thus $\bar{P}_{s_{2}} \approx \bar{P}_{s_{1}}$. Whereas, as $M=2,4$, we obtain $13 \xi / 48 \rho \ll \omega$, leading to $\bar{P}_{s_{2}} \gg P_{s_{1}}$. This concludes the proof.

It is worth noting from Theorem 1 that in MCIK-OFDM with large $M$, the GD detector is preferred to the ML since the GD offers the nearly optimal error performance at much lower complexity. Notice that the complexities of the GD and the ML are $\mathcal{C}_{G D}=N+2 K M$ and $\mathcal{C}_{M L}=2^{p_{1}+1} M^{K}$, respectively [17]. As $K$ and $M$ increase, we obtain $\mathcal{C}_{M L} \gg \mathcal{C}_{G D}$.

\section{B. Fixed CSI Uncertainty, $\left(\epsilon^{2}>0\right)$}

For given $\epsilon^{2}>0$, as $\bar{\gamma}$ increases, (17) and (20) can be asymptotically approximated by

$$
\begin{aligned}
& \bar{P}_{s_{1}} \approx \underbrace{\frac{\tilde{\Psi}_{1}}{12}\left[\left(1+\frac{1-\epsilon^{2}}{2 \epsilon^{2}}\right)^{-2}+3\left(1+\frac{2-2 \epsilon^{2}}{3 \epsilon^{2}}\right)^{-2}\right]}_{A_{1}} \\
& +\underbrace{\frac{\Psi_{2}}{12}\left[\frac{1}{1+\frac{\left(1-\epsilon^{2}\right) \rho}{\epsilon^{2}}}+\frac{3}{1+\frac{4\left(1-\epsilon^{2}\right) \rho}{3 \epsilon^{2}}}\right]}_{A_{2}} \\
& \bar{P}_{s_{2}} \approx \frac{\Psi_{2}}{12}\left[\frac{1}{1+\frac{\left(1-\epsilon^{2}\right) \rho}{\epsilon^{2}}}+\frac{3}{1+\frac{4\left(1-\epsilon^{2}\right) \rho}{3 \epsilon^{2}}}\right],
\end{aligned}
$$

which no longer rely on the SNRs, i.e., $\bar{\gamma}$ or $\gamma_{0}$.

It is worth noting that for fixed $\epsilon^{2}$, the SEP at high SNRs achieves zero diversity and coding gains for both the ML and GD detectors. This is because there exists an error floor on the average SEP so that increasing the SNR does not improve the error performance. In addition, the average SEP of the GD with fixed $\epsilon^{2}$ is not affected by $N$ at high SNRs since $\Psi_{2}$ does not depend on $N$.

For the comparison between the ML and GD in terms of the SEP, we obtain an interesting theorem as follows.

Theorem 2. Under fixed CSI uncertainty, in MCIK-OFDM, the low-complexity GD detector provides a better error performance than the ML detector at high SNRs, i.e., $\bar{P}_{s_{1}}>\bar{P}_{s_{2}}$.

Proof: It is shown from (28) and (29) that $\bar{P}_{s_{1}} \approx$ $A_{1}+A_{2}>\bar{P}_{s_{2}} \approx A_{2}$, where $A_{1}$ and $A_{2}$ are related to the asymptotic IEP of the ML and the SEP of $M$-ary symbols, respectively. This concludes the proof.

Remark 4. Under the fixed channel uncertainty, the GD outperforms the ML in terms of both the computational complexity and the error performance. This is totally contrary to the behavior of the SEP in perfect CSI, where the GD is always worse than the ML in terms of the SEP.

In the next subsection, we consider another scenario when $\epsilon^{2}$ varies as a decreasing function of the SNR, where the MMSE estimator is a good and practical example.

\section{MMSE Based Variable CSI Uncertainty}

Based on the MMSE principle in the channel estimation, we have

$$
\epsilon^{2}=\frac{1}{1+E_{s} / N_{0}}
$$

which is proved in Appendix B. It is clearly shown in (30) that the MMSE error variance gets smaller as the SNR gets larger. Inserting (30) into (17) and (20), at high SNRs, we attain

$$
\begin{gathered}
\bar{P}_{s_{1}} \approx \frac{13 \xi K(N+K)}{48 \rho N(K+1)}\left(\frac{1}{\gamma_{0}}\right), \\
\bar{P}_{s_{2}} \approx \frac{K}{N(K+1)}\left[K \omega+\frac{13 \xi(N+K)}{48 \rho}\right]\left(\frac{1}{\gamma_{0}}\right) .
\end{gathered}
$$

The corresponding coding gains are

$$
\begin{gathered}
c_{1}^{\prime}=\frac{48 \rho N(K+1)}{13 \xi K(N+K)}, \\
c_{2}^{\prime}=\frac{N(K+1)}{K\left[K \omega+\frac{13 \xi(N+K)}{48 \rho}\right]} .
\end{gathered}
$$

Particularly, as $K \rightarrow N$, (33) can reduce to the coding gain of the classical OFDM, as

$$
c_{0}^{\prime}=\frac{24 \rho}{13 \xi} .
$$

Like the perfect CSI case, it can be seen from (31) and (32) that the MCIK-OFDM scheme under MMSE based $\epsilon^{2}$ achieve the diversity order of one. However, for high SNR, the attainable coding gains get smaller, i.e., $c_{i}^{\prime}<c_{i}$ for $i=0,1,2$. This degradation is caused by the impact of MMSE channel estimation error. Based on this, we provide the following theorem.

Theorem 3. Consider MCIK-OFDM systems. Denote by $\Delta_{1}$ and by $\Delta_{2}$ coding gain losses achieved by the $M L$ and by the $G D$, respectively, under MMSE based uncertain CSI over the perfect CSI. Then, we have

$$
\begin{gathered}
\Delta_{1}=10 \log _{10}\left(\frac{N+K}{K}\right), \\
\Delta_{2}=10 \log _{10}\left[\frac{K \omega+\frac{13 \xi(N+K)}{48 \rho}}{K \omega+\frac{13 \xi K}{48 \rho}}\right],
\end{gathered}
$$

which satisfy $\Delta_{1}>\Delta_{2}$.

Proof: Let the losses of coding gain be $\Delta_{i}=$ $10 \log _{10}\left(c_{i} / c_{i}^{\prime}\right)$ for $i=1,2$. Thus, utilizing (24) and (33) yields (36), while using (25) and (34) yields (37).

Subtracting (37) from (36) leads to

$$
\Delta_{1}-\Delta_{2}=10 \log _{10}\left\{1+\frac{N K \omega}{K\left[K \omega+\frac{13 \xi(N+K)}{48 \rho}\right]}\right\}>0 .
$$


This concludes the proof.

Remark 5. As shown in Theorem 3, the GD detector is less sensitive to the MMSE channel estimation error than the ML. This is because the loss of coding gain of the ML is larger than that of the GD.

Theorem 4. Denote by $g^{\prime}$ the coding gain that the MCIKOFDM using the ML achieves over the classical OFDM, under MMSE CSI uncertainty. We have

$$
g^{\prime}=10 \log _{10}\left[\frac{2 N(K+1)}{K(N+K)}\right](\mathrm{dB}),
$$

and $g^{\prime}<g$, where $g$ is given in (27).

Proof: Based on the definition of $g^{\prime}$, we have $g^{\prime}=$ $10 \log _{10}\left(c_{1}^{\prime} / c_{0}^{\prime}\right)$. Here, using $c_{1}^{\prime}, c_{0}^{\prime}$ given in (33) and (35), respectively, we obtain (38). Next, combining (27) and (38) leads to $g-g^{\prime}=10 \log _{10}[(N+K) / 2 K]>0$ for every $K<N$, thus $g^{\prime}<g$. This concludes the proof.

Remark 6. Theorem 4 reveals that under MMSE CSI uncertainty, the coding gain achieved by MCIK-OFDM over the classical OFDM is smaller than that under the perfect CSI.

Finally, similar to Theorem 1 in the perfect CSI case, in this case, the SEP of the GD performs nearly the same as that of the ML as M increases, which can be shown from (31) and (32) with the steps used in Theorem 1.

\section{NUMERICAL AND SIMULATION RESULTS}

We now present numerical and simulation results to verify our theoretical analysis on the SEP of the MCIK-OFDM under various CSI conditions. For comparison, we show the average SEP for MCIK-OFDM schemes using the ML and the GD as well as the classical OFDM.

\section{A. SEP of MCIK-OFDM under Perfect CSI}

Fig. 1 shows the average SEP of MCIK-OFDM with $N=$ 4, $K=2, M=2$, using both the ML and the GD. This figure also compares this MCIK-OFDM scheme with the classical $\mathrm{OFDM}$ at the same data rate of $1 \mathrm{bps} / \mathrm{Hz}$. As seen from Fig. 1 , both the theoretical and asymptotic curves are very tight to simulation results in a wide range of SNRs. This successfully verifies the accuracy of the derived expressions for the average SEPs of both the ML and GD. Moreover, the SEP of MCIKOFDM and the classical OFDM achieve the same diversity order of one, under perfect CSI, as shown in Remark 1.

Fig. 2 illustrates the impact of the PSK modulation order $M$ on the average IEP of MCIK-OFDM using the ML with $N=$ $4, K=2$ and $M=\{2,4,8,16\}$. It is clear from Fig. 2 that the order $M$ has a negligible impact on the IEP performance, which validates our analysis right after (16). We also see the tightness of the derived IEP, even at low SNRs.

Fig. 3 depicts the behaviour of the SEP of MCIK-OFDM using the ML and the GD at various $K$. It can be seen that MCIK-OFDM with the ML and $N=4, M=2$ provides the difference of $6.5 \mathrm{~dB}$ SNR gain when increasing $K$ from 1 to 3. However, greater $K$ offers a higher data rate. In addition, Fig. 3 shows that compared to OFDM, the SNR gain of the $K=1$ MCIK-OFDM with the ML achieves about $8 \mathrm{~dB}$ SNR gain for the SEP of $10^{-3}$. This validates Remark 3 .

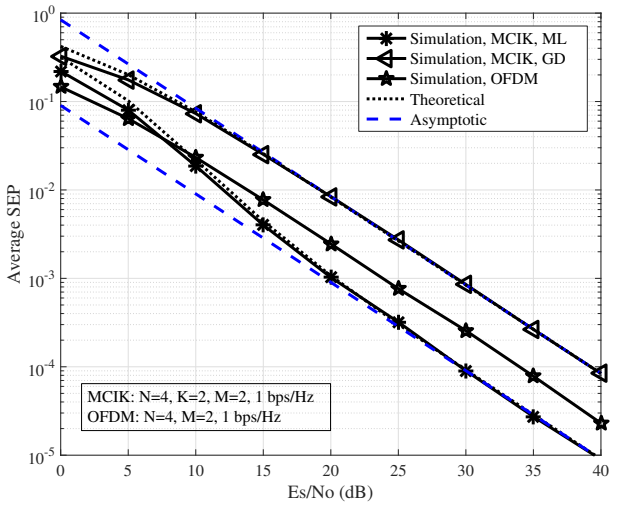

Fig. 1. Average SEPs of MCIK-OFDM with the ML and the GD, and comparison with OFDM when $N=4, K=2$ and $M=2$, at $1 \mathrm{bps} / \mathrm{Hz}$.

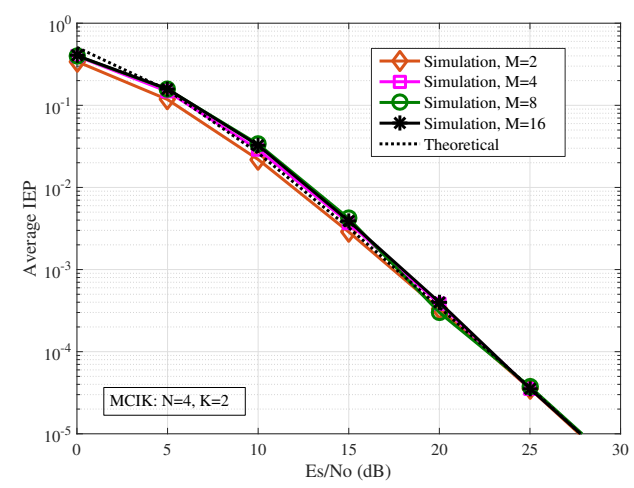

Fig. 2. The effect of $M$ on the average IEP of MCIK-OFDM using the ML with $N=4, K=2$ and $M=\{2,4,8,16\}$.

In Fig. 4, we compare the average SEPs of two detectors in MCIK-OFDM with various $M$. This figure shows that for $M=2$, the ML significantly outperforms the GD, with an SNR gain of approximately $8 \mathrm{~dB}$ at high SNRs. Whereas, as $M$ increases to 8 or 16 , the SEP of the GD approaches to that of the ML. Especially when $M=16$, the performance gap between the two detectors is negligible. This validates Theorem 1. In addition, this figure confirms the accuracy of the asymptotic SEPs, especially at high SNRs.

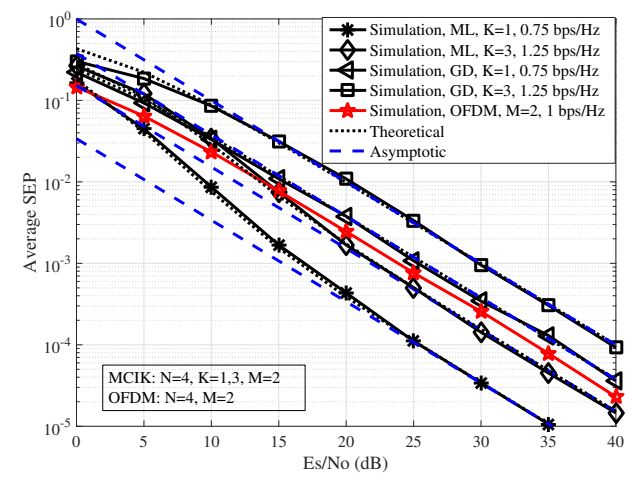

Fig. 3. Impact of $K$ on the average SEP of MCIK-OFDM using both the ML and GD when $N=4, K=\{1,3\}$ and $M=2$. 


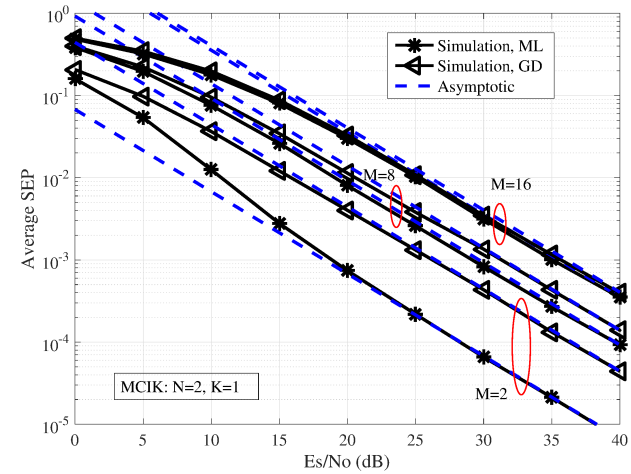

Fig. 4. Comparison between the average SEPs of the ML and the GD, with the MCIK-OFDM of $N=2, K=1$ and $M=\{2,8,16\}$.

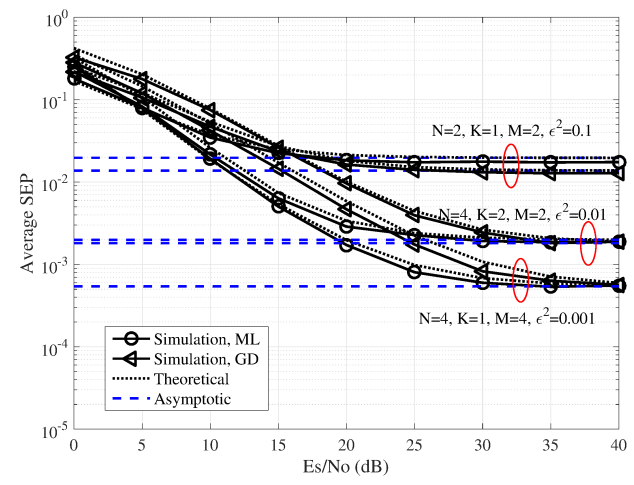

Fig. 5. Impact of fixed $\epsilon^{2}$ on the average SEPs of different MCIK-OFDM schemes using both the ML and GD when $N=\{2,4\}, K=\{1,2\}, M=$ $\{2,4\}$, and $\epsilon^{2}=\{0.1,0.01,0.001\}$.

\section{B. SEP of MCIK-OFDM under Fixed CSI Uncertainty}

Fig. 5 illustrates the average SEPs of a number of MCIKOFDM schemes using both the ML and GD detectors, under different values of $\epsilon^{2}$. It is shown from Fig. 5 that the average SEPs suffer from error foors as the SNR increases. These error floors get higher when increasing $\epsilon^{2}$. More interestingly, as $\epsilon^{2}=0.1$, the GD outperforms the ML in terms of the SEP, especially at high SNRs. This observation validates the accuracy of Theorem 2 and Remark 4. As $\epsilon^{2}$ decreases, the SEP gap between the two detectors becomes smaller.

\section{SEP of MCIK-OFDM under MMSE CSI Uncertainty}

Fig. 6 shows the effect of the MMSE imperfection on the SEP of MCIK-OFDM with the ML and GD, when $N=4, K=2$ and $M=2$. As seen from Fig. 6, under MMSE CSI uncertainty, MCIK-OFDM using the ML suffers from a considerable loss of nearly $5 \mathrm{~dB}$ SNR gain compared to the perfect CSI case. The loss raised by the GD is much smaller, being around $0.5 \mathrm{~dB}$. Thus, the GD is less sensitive to MMSE CSI uncertainty than the ML.

In Fig. 7, we depict the average SEP of MCIK-OFDM using the ML with $N=4, K=3, M=4$ under MMSE imperfection. For comparison, the classical OFDM with $N=4, M=4$ is also depicted. As seen from Fig. 7, under perfect CSI, MCIK-OFDM can remarkably outperform OFDM in terms of the SEP. However, this is no longer true under MMSE CSI

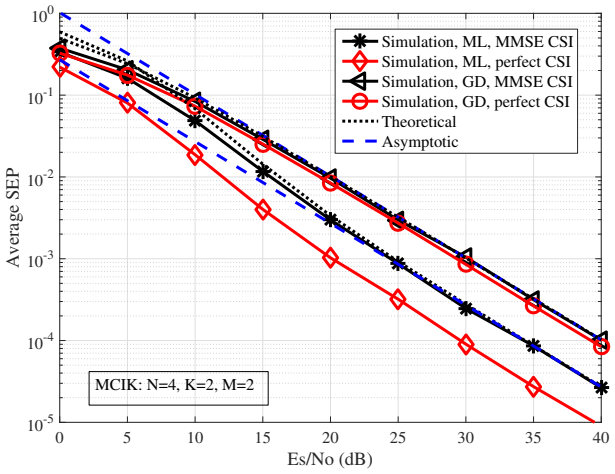

Fig. 6. Impact of MMSE based CSI uncertainty on the average SEPs of MCIK-OFDM using both the ML and GD when $N=4, K=2$ and $M=2$.

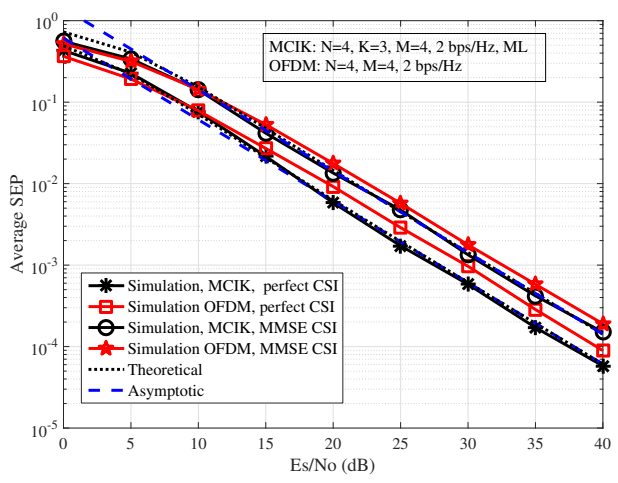

Fig. 7. SEP comparison between MCIK-OFDM using the ML and OFDM when $N=4, K=3$ and $M=4$ under MMSE varible and perfect CSI.

uncertainty, where the SEP of MCIK-OFDM is slightly better than that of the OFDM.

\section{CONCLUSIONS}

We have proposed a novel framework to analyze the average SEPs of the generalized MCIK-OFDM with any types of detector, taking into account CSI imperfection. The accurate, closed-form expressions for the SEPs of MCIK-OFDM using the ML and the GD are derived, in the presence of CSI uncertainty. We investigated asymptotic SEPs with respect to the three CSI conditions including perfect, fixed and variable MMSE based CSI uncertainties. This ensured to provide a comprehensive insight into the diversity and coding gains achieved by MCIK-OFDM with the ML and GD, even under various CSI conditions. Interestingly, our results clearly showed that the GD is not only less sensitive to channel estimation errors such as the MMSE CSI imperfection, but also can outperform the ML in terms of the SEP under the fixed CSI conditions. We have clearly shown that the GD can be promising to offer the best or near optimal SEP under uncertain CSI. The derived closed-form SEP expressions can be useful for the design of various MCIK-OFDM systems.

\section{APPENDIX A \\ PROOF OF LEMMA 1}

The received signal at the active sub-carrier index $\alpha$ is given by $y(\alpha)=x(\alpha) \hat{h}(\alpha)+\tilde{n}(\alpha)$, where $\tilde{n}(\alpha)=$ 
$x(\alpha) e(\alpha)+n(\alpha)$ is the noise caused by the CSI uncertainty and the additive noise, being $\mathcal{C N}\left(0, \varphi E_{s} \epsilon^{2}+N_{0}\right)$. Therefore, the ML detection per sub-carrier will detect $x(\alpha)$ with an instantaneous SNR of $\hat{\gamma}_{\alpha}=\varphi E_{s}|\hat{h}(\alpha)|^{2} /\left(\varphi E_{s} \epsilon^{2}+N_{0}\right)=$ $\bar{\gamma} \hat{\nu}_{\alpha} /\left(1+\bar{\gamma} \epsilon^{2}\right)$, where $\hat{\nu}_{\alpha}=|\hat{h}(\alpha)|^{2}$. According to [25], a well-known approximation of $P_{M}(\alpha)$ is given as

$$
P_{M}(\alpha) \approx \xi Q\left[\sqrt{\frac{2 \bar{\gamma} \hat{\nu}_{\alpha}}{1+\bar{\gamma} \epsilon^{2}}} \sin \left(\frac{\pi}{M}\right)\right]
$$

where $\xi=1$ for $M=2$ and $\xi=2$ for $M>2$. Using the approximation of Q-function of $Q(x) \approx \frac{1}{12} e^{-x^{2} / 2}+\frac{1}{4} e^{-2 x^{2} / 3}$, $P_{M}(\alpha)$ can be represented by

$$
P_{M}(\alpha) \approx \frac{\xi}{12}\left(e^{-\frac{\bar{\gamma} \hat{\nu} \alpha}{1+\bar{\gamma} \epsilon^{2}}}+3 e^{-\frac{4 \bar{\gamma} \hat{\nu} \alpha}{3+3 \bar{\gamma} \epsilon^{2}}}\right),
$$

where $\rho=\sin ^{2}(\pi / M)$.

Note that the MGF of $\hat{\nu}_{\alpha}$ is given by $\mathcal{M}_{\hat{\nu}}(z)=$ $\left[1-\left(1-\epsilon^{2}\right) z\right]^{-1}$. Based on the definition of MGF function, i.e., $\mathbb{E}_{\hat{\nu}}\left\{e^{z \hat{\nu}}\right\}=\mathcal{M}_{\hat{\nu}}(z)$, the average SEP of the $M$-ary symbol detection is obtained as (11). This concludes the proof.

\section{APPENDIX B \\ DERIVATION OF (30)}

For all sub-carriers, assume that the MMSE estimator uses the unit pilot signal, i.e., $x_{p}(\alpha)=1$, which is transmitted with the power $E_{s}$. The received pilot signal at each sub-carrier is $y(\alpha)=\sqrt{E_{s}} h(\alpha)+n(\alpha)$, which is used to estimate the channel coefficient as

$\hat{h}(\alpha)=\frac{y \mathbb{E}\left\{y(\alpha) h^{*}(\alpha)\right\}}{\mathbb{E}\left\{|y(\alpha)|^{2}\right\}}=\frac{\sqrt{E_{s}}}{E_{s}+N_{0}}\left[\sqrt{E_{s}} h(\alpha)+n(\alpha)\right]$

As a result, the channel estimation error per sub-carrier is

$$
e(\alpha)=h(\alpha)-\hat{h}(\alpha)=\frac{N_{0}}{E_{s}+N_{0}} h(\alpha)-\frac{\sqrt{E_{s}}}{E_{s}+N_{0}} n(\alpha),
$$

From (41), we easily obtain (30). This concludes the proof.

\section{REFERENCES}

[1] R. Abu-alhiga and H. Haas, "Subcarrier-index modulation OFDM," in Proc. IEEE Pers., Indoor., Mobile Radio Commun., Sept 2009, pp. 177181 .

[2] E. Basar, U. Aygolu, E. Panayirci, and H. V. Poor, "Orthogonal frequency division multiplexing with index modulation," IEEE Trans. Signal Process., vol. 61, no. 22, pp. 5536-5549, Nov 2013.

[3] Y. Ko, "A tight upper bound on bit error rate of joint OFDM and multicarrier index keying," IEEE Commun. Lett., vol. 18, no. 10, pp. 17631766, Oct 2014

[4] M. Wen, X. Cheng, M. Ma, B. Jiao, and H. V. Poor, "On the achievable rate of OFDM with index modulation," IEEE Trans. Signal Process., vol. 64, no. 8, pp. 1919-1932, April 2016.

[5] R. Fan, Y. J. Yu, and Y. L. Guan, "Generalization of orthogonal frequency division multiplexing with index modulation," IEEE Trans. Wireless Commun., vol. 14, no. 10, pp. 5350-5359, Oct 2015.

[6] J. Crawford and Y. Ko, "Low complexity greedy detection method with generalized multicarrier index keying OFDM," in Proc. IEEE Pers., Indoor, Mobile Radio Commun., Aug 2015, pp. 688-693.

[7] E. Chatziantoniou, J. Crawford, and Y. Ko, "Performance analysis of a low-complexity detector for MCIK-OFDM over TWDP fading," IEEE Commun. Lett., vol. 20, no. 6, pp. 1251-1254, June 2016.
[8] E. Basar, "OFDM with index modulation using coordinate interleaving," IEEE Wireless Commun. Lett., vol. 4, no. 4, pp. 381-384, Aug 2015.

[9] J. Zheng and R. Chen, "Achieving transmit diversity in OFDM-IM by utilizing multiple signal constellations," IEEE Access, vol. PP, no. 99, pp. 1-1, 2017.

[10] M. Wen, B. Ye, E. Basar, Q. Li, and F. Ji, "Enhanced orthogonal frequency division multiplexing with index modulation," IEEE Trans. Wireless Commun., vol. PP, no. 99, pp. 1-1, 2017.

[11] J. Choi, "Coded OFDM-IM with transmit diversity," IEEE Trans. Commun., vol. PP, no. 99, pp. 1-1, 2017.

[12] B. Zheng, F. Chen, M. Wen, F. Ji, H. Yu, and Y. Liu, "Lowcomplexity ML detector and performance analysis for OFDM with in-phase/quadrature index modulation," IEEE Commun. Lett., vol. 19, no. 11, pp. 1893-1896, Nov 2015.

[13] T. Mao, Z. Wang, Q. Wang, S. Chen, and L. Hanzo, "Dual-mode index modulation aided OFDM," IEEE Access, vol. 5, pp. 50-60, 2017.

[14] M. Wen, E. Basar, Q. Li, B. Zheng, and M. Zhang, "Multiple-mode orthogonal frequency division multiplexing with index modulation," IEEE Trans. Commun., vol. PP, no. 99, pp. 1-1, 2017.

[15] E. Basar, "On multiple-input multiple-output OFDM with index modulation for next generation wireless networks," IEEE Trans. Signal Process., vol. 64, no. 15, pp. 3868-3878, Aug 2016.

[16] B. Zheng, M. Wen, E. Basar, and F. Chen, "Multiple-input multipleoutput OFDM with index modulation: Low-complexity detector design,' IEEE Trans. Signal Process., vol. 65, no. 11, pp. 2758-2772, June 2017.

[17] J. Crawford, E. Chatziantoniou, and Y. Ko, "On the SEP analysis of OFDM index modulation with hybrid low complexity greedy detection and diversity reception," IEEE Trans. Veh. Technol., vol. PP, no. 99, pp. 1-1, 2017.

[18] N. Ishikawa, S. Sugiura, and L. Hanzo, "Subcarrier-index modulation aided OFDM - will it work?" IEEE Access, vol. 4, pp. 2580-2593, 2016.

[19] E. Basar, "Index modulation techniques for 5G wireless networks," IEEE Commun. Mag., vol. 54, no. 7, pp. 168-175, July 2016.

[20] Q. Ma, P. Yang, Y. Xiao, H. Bai, and S. Li, "Error Probability Analysis of OFDM-IM With Carrier Frequency Offset," IEEE Commun. Lett., vol. 20, no. 12, pp. 2434-2437, Dec 2016.

[21] M. Wen, Y. Zhang, J. Li, E. Basar, and F. Chen, "Equiprobable Subcarrier Activation Method for OFDM With Index Modulation," IEEE Commun. Lett., vol. 20, no. 12, pp. 2386-2389, Dec 2016.

[22] A. I. Siddiq, "Effect of Subcarrier Activation Ratio on the Performance of OFDM-IM over Rayleigh Fading Channel," IEEE Commun. Lett., vol. 21, no. 6, pp. 1293-1296, June 2017.

[23] G. Kaddoum, Y. Nijsure, and H. Tran, "Generalized code index modulation technique for high-data-rate communication systems," IEEE Trans. Veh. Technol., vol. 65, no. 9, pp. 7000-7009, Sept 2016.

[24] E. Soujeri, G. Kaddoum, M. Au, and M. Herceg, "Frequency index modulation for low complexity low energy communication networks," IEEE Access, vol. PP, no. 99, pp. 1-1, 2017.

[25] J. Proakis, Digital Communications. McGraw-Hill, 2001.

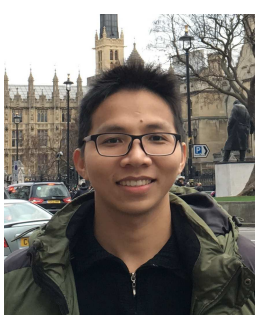

Thien Van Luong received the B.S degree in electrical engineering from Hanoi University of Science and Technology, Vietnam, in 2015. He is currently pursuing the Ph.D. degree with Queen's University Belfast, Northern Ireland. In 2016, he was a research assistant of Wireless Networks and Decision Systems (WNDS) Group, Singapore University of Technology and Design. His research is in the areas of index modulation, spatial modulation, and their applications in machine type communications. 


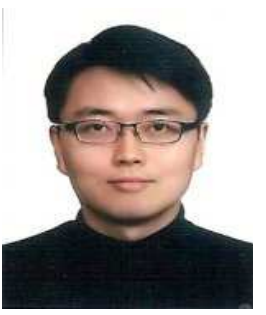

Youngwook Ko received Ph.D. in 2006 and M.S. in 2002, in Electrical Engineering from Arizona State University, Tempe, AZ USA, and B.S.E. in Information and Communications Engineering from Hannam University, South Korea. After his Ph.D., he worked at several places in both industry and academia. Since 2013, Dr. Ko has been with the ECIT Institute at the Queen's University of Belfast as a Lecturer in Wireless Innovation Centre. He has authored over 40 publications in major IEEE international journals, and peer-reviewed international conferences. Dr Ko is the recipient of several EPSRC and Newton projects. He is on the Editorial Board of the Elsevier Journal on Physical Communications and a member of the EPSRC Peer Review Associate College. His research is in the areas of multicarrier index keying, machine type communications, and industrial IoT for the next generation wireless communications. 